\title{
Further Studies on a Novel Animal Model of Depression: Therapeutic Effects of a Tricyclic Antidepressant
}

\author{
K. A. ROTH \\ Nancy Pritzker Laboratory of Behavioral Neurochemistry \\ Department of Psychiatry and Behavioral Sciences \\ Stanford University School of Medicine, Palo Alto, CA 94305
}

AND

R. J. KATZ ${ }^{1}$

Mental Health Research Institute, Department of Psychiatry

University of Michigan Medical Center, Ann Arbor, MI 48109

Received 15 February 1980

\begin{abstract}
ROTH, K. A. AND R. J. KATZ. Further studies on a novel animal model of depression: Therapeutic effects of a tricyclic antidepressant. NEUROSCI. BIOBEHAV. REV. 5(2) 253-258, 1981.-We have previously shown that rats given stress before open field testing have elevated activity in comparison with unstressed rats. This acute behavioral response may be eliminated by chronic stress and restored by pretreatment with the monoamine oxidase inhibitor pargyline. The present study replicated previous findings upon the effects of acute and chronic stress and extended results upon treatment to the class of tricyclic antidepressant drugs, using imipramine as a prototypic tricyclic antidepressant. Imipramine also restored both behavioral and psychoendocrine activity which was otherwise altered by chronic stress.
\end{abstract}

Activity Ambulation Defecation Depression Imipramine Open field

IN previous reports we have argued that the development of novel preclinical models of depression remains an important task in the eventual understanding of clinical disorders [5]. We also have described a novel procedure by which a state of relative behavioral depression may be induced in the rat $[6,7]$. The model is similar to learned helplessness [12], behavioral despair [9], and other models (e.g. [8]). The depression model utilizes acute behavioral responses to stress in an open field following either chronic stress or control treatment, and was shown to share both endocrine and psychopharmacological similarities to endogenous depression. Pharmacological similarity was established based upon reversal of otherwise present depression by the monoamine oxidase inhibitor pargyline. A second and more widely employed clinical therapy for unipolar depression involves tricyclic antidepressants, and imipramine is a prototypic drug of this class $[1,11]$.

The present experiment both replicated selected findings from previous studies and extended the range of compounds tested in the stress-depression model to the class of tricyclics.

\section{METHOD}

Subjects. Sixty-nine adult male Sprague-Dawley rats (Charles River Farms, Portage, MI), 70 days on the day of testing, were housed $2 /$ cage in $25 \times 18 \times 17 \mathrm{~cm}$ stainless steel cages. Food (Teklad $4.0 \%$ fat rodent diet S-0836) and tap water were available ad lib for control and experimental groups except for three occasions when food or water was removed from the experimental groups for $16 \mathrm{hr}$ as part of the chronic stress procedure. Lighting cycles of $12 \mathrm{hr} / 12 \mathrm{hr}$ (lights on from 7:00 to 19:00 hr) were automatically programmed. Rats were housed as described for 3 weeks prior to testing.

Apparatus. Testing was carried out in a square white Plexiglas open field each side of which was $1.22 \mathrm{~m}$ and the height of which was $45 \mathrm{~cm}$. The apparatus was divided into 16 equal shares $30.5 \mathrm{~cm} /$ side to allow for the assessment of locomotion. The open field was thoroughly cleaned between tests.

Behavioral procedure. A partial factorial design was utilized for open field testing. The first factor was presence

\footnotetext{
'Correspondence to Dr. Katz at Department of Psychology, Johns Hopkins University, Charles and 34th Street, Baltimore, MD 21218.
} 
or absence of an immediately antecedent noise-light stress. This factor was varied across three conditions. A control group received no additional manipulations; a second group received exposure to a chronic stress procedure (described below) and daily injections of $0.9 \%$ saline; a third group was exposed to the identical stress procedure as group two and received daily injections of the tricyclic antidepressant imipramine $(5 \mathrm{mg} / \mathrm{kg} /$ day) for 15 days. Additional rats from each group were sacrificed without exposure to the open field to obtain basal corticosterone levels. An explicit control for the effects of imipramine upon acute stress was not included.

It should be noted this clearly represents a critical comparison, and that antidepressants do not generally facilitate the behavioral effects of acute noise stress. Because the issue is important and to allow an adequate comparative investigation a separate set of studies (to be subsequently reported) upon acute stress interactions was carried out.

\section{First Factor: Acute Stress Procedure}

Animals were habituated to the presence of an experimenter during the dark cycle. During the week prior to testing, laboratory personnel entered the housing room and performed routine tasks at the time when testing was to be later carried out (between 2100 and $2400 \mathrm{hr}$ ).

On the test day both subjects in a cage were removed and placed individually in $48 \times 27 \times 20 \mathrm{~cm}$ polypropylene cages (Scientific Products Series 140) for transport. One rat was immediately taken $6 \mathrm{~m}$ down an unlit corridor to the test room and placed in the open field for testing. The second rat was taken a similar distance to a brightly lit room $(8 \times 70 \mathrm{~W}$ lamps) and placed $1 \mathrm{~m}$ from a speaker emitting a $95 \mathrm{~dB}$ white noise. After one hour exposure to the noise-light stress, the acutely stressed rat was transported to the test room and placed in the open field for testing.

\section{Second Factor: Chronic Stress Procedure}

Thirteen stressors were administered over a fifteen day period. Stressors and times of delivery were varied so as to maximize the unpredictable nature of the stress procedure. The following stressors were used: switch cage mates (two times), $24 \mathrm{hr}$ isolation, $30 \mathrm{~min}$ of unpredictable shock (one $1.0 \mathrm{~mA}$ shock $/ 60 \mathrm{sec}$ ) (two times), $16 \mathrm{hr}$ water deprivation (two times), $16 \mathrm{hr}$ food deprivation, $5 \mathrm{~min}$ swim in $4.0^{\circ} \mathrm{C}$ ice water (two times), 60 min of horizontal shaker (two times), and $1 \mathrm{~min}$ tail pinch. These were administered every day to two days throughout the lighting cycle (0600 to 1900). Table 1 presents the schedules of stress delivery.

\section{Testing Procedure}

For all subjects the test room was illuminated by six GE F96712R fluorescent lights which provided dim (500 mphot) red light $(600-700 \mathrm{~nm})$ that was subliminal for the subjects but allowed experimental observation. A continuous background masking noise of 40-50 dB was provided by normal operation of the air circulation system.

Subjects were placed in a corner of the open field facing the apparatus wall and a twelve minute observation period was automatically timed. The test interval was extended in comparison with the previous experiment due to reduced availability of laboratory personnel. In all cases, however, the final six minute scores were discarded to allow comparison with previous data and the experiments to be subsequently reported. In addition, for two measures the first 3
TABLE 1

STRESS PROCEDURE

\begin{aligned} & \hline Day \multicolumn{1}{c}{ Treatment } \\ & \hline 1 ear mark \\ & 2 shaker stress (high speed $1 \mathrm{hr}) \\ & 3$ cold swim $\left(4^{\circ} \mathrm{C}-5 \mathrm{~min}\right) \\ & 4$ switch cage mates \\ & 5 shock $(1 / 2 \mathrm{hr}) \\ & 6$ remove water $(16 \mathrm{hr}) \\ & 7$ tail pinch \\ & 8 remove food $(16 \mathrm{hr}) \\ & 9$ shock $(1 / 2 \mathrm{hr}) \\ & 10$ remove water $(16 \mathrm{hr}) \\ & 11$ individual housing $(24 \mathrm{hr}) \\ & 12$ coid swim \\ & 13 shaker stress \\ & 14 switch cage mates \\ & 15 test \\ & \hline\end{aligned}

minutes were taken as the primary datum. Again, this was done to allow comparison with previous studies from our and other laboratories. The following measures were recorded: defecation score (boluses/session), latency to initial defecation (in sec), latency to leave home square (in sec), outside squares crossed (in $3 \mathrm{~min}$ ), inside square crossed, and number of rears (in $6 \mathrm{~min}$ ). At the end of testing, subjects were immediately removed, sacrificed by decapitation, and trunk blood was collected in heparinized tubes. Following centrifugation at $2400 \mathrm{rpm}$ for $30 \mathrm{~min}$, plasma was collected and frozen at $-40^{\circ} \mathrm{C}$ for later corticosterone determination, using the method of Murphy [10]. All determinations were performed in duplicate using rat corticosterone as the assay standard. The coefficient of within assay variation was less than $5 \%$, and did not exceed $8 \%$ across assays.

In addition to direct behavioral measures (described above) a derived composite measure of activation was calculated based upon an average of ranks across categories. Groups were rank ordered from lowest (1) to highest (6) on all measures except on initial latency to leave home square and defecation score, for which reverse orders were used. A mean score (average across measures) was then computed for each group across ranks.

\section{RESULTS}

Acute stress increased a number of motor behaviors and decreased defecation in comparison with controls. Chronic stress plus vehicle treatment eliminated the behavioral activation seen in control rats after acute noise-light stress. Treatment of chronically stressed rats with imipramine restored the acute activation response to the noise-light stress.

These findings were confirmed statistically, and are presented in Figs. 1 through 7. Figure 1 presents data based upon outside squares crossed ( $0-3$ minutes). There were significant differences across categories, $F(5,42)=3.5$, $p<0.01$. Of interest are the following significant differences: $(p<0.05$ in all cases by Scheffe's test: this criterion is used throughout). Acute stress elevated initial activity in comparison to an unstressed control; the chronic stress saline 


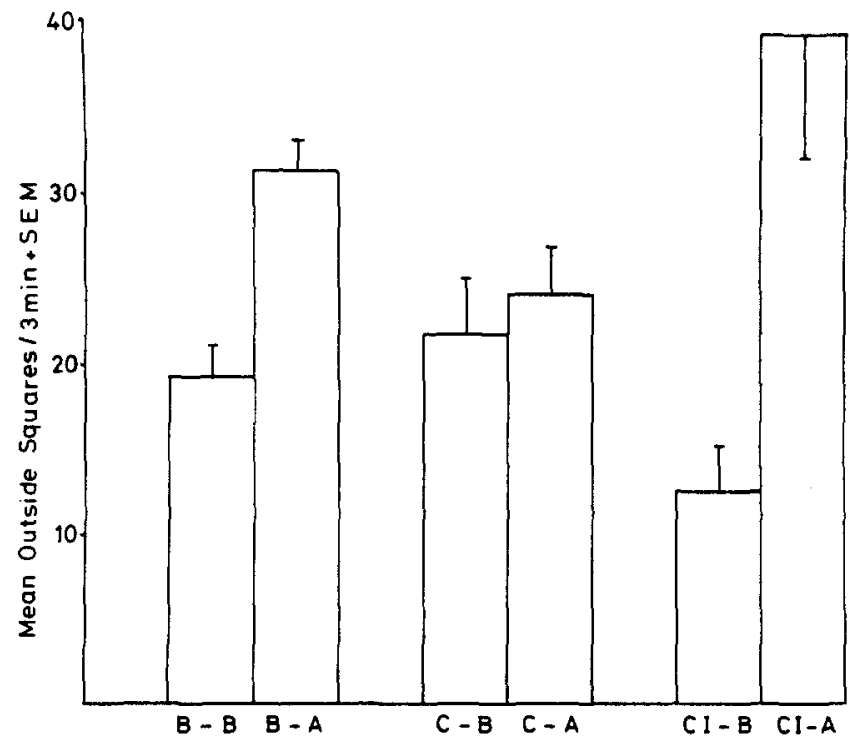

FIG. 1. Effects of acute and chronic stress and imipramine upon open field activity in the rat (mean outside squares/3 min +SEM). $B=$ basal, $A=$ acute stress, $C=$ chronic stress, $I=$ chronic imipramine pretreatment $(5 \mathrm{mg} / \mathrm{kg} / \mathrm{day})$. For all cases the leftmost of the two columns displays basal (i.e., no acute stress) activity and the rightmost shows behavioral response to acute stress. Scores reflect grid crossings of animals upon initial exposure to an open field.

group did not show an elevation in activity after acute stress; drug treatment apparently reversed this loss of response to acute stress. The imipramine treated chronically stressed group receiving acute stress was not reliably different from the nonchronic (i.e., control) group after similar treatment. This general pattern supports the postulated model and was confirmed by other measures.

Latency to leave home square scores reflected an overall change, $\mathrm{F}(5,42)=2.62, p<0.05$ (Fig. 2). This was due to reductions brought about by acute stress across conditions. Again the acutely stressed group moved more quickly than its control; the chronically stressed saline group showed a marginally significant activation compared to its unstressed control $(p<0.09)$. Due to a higher baseline imipramine treatment restored the acute activation response compared to its unstressed control, but not significantly. It must be emphasized that the latency measure did not reflect major alterations brought about by chronic stress.

Center field penetration followed a similar course (Fig. 3). However, the difference between groups was not statistically significant, $F(5,42)=1.4, p=0.3$. Further analysis was therefore precluded.

Rearing scores are presented in Fig. 4. An overall effect of groups was observed, $F(5,42)=3.4, p<0.01$. However the only significant increase in comparison with a basal (i.e., nonacutely stressed control was in nonchronically stressed rats, $\mathrm{F}=4.8 ; p<0.03$. No acute elevation was seen in chronically stressed rats without imipramine, nor was an effect seen in the imipramine treated animals, rather the drug appeared nonspecifically excitatory.

Defecation scores (Fig. 5) followed a pattern converse to

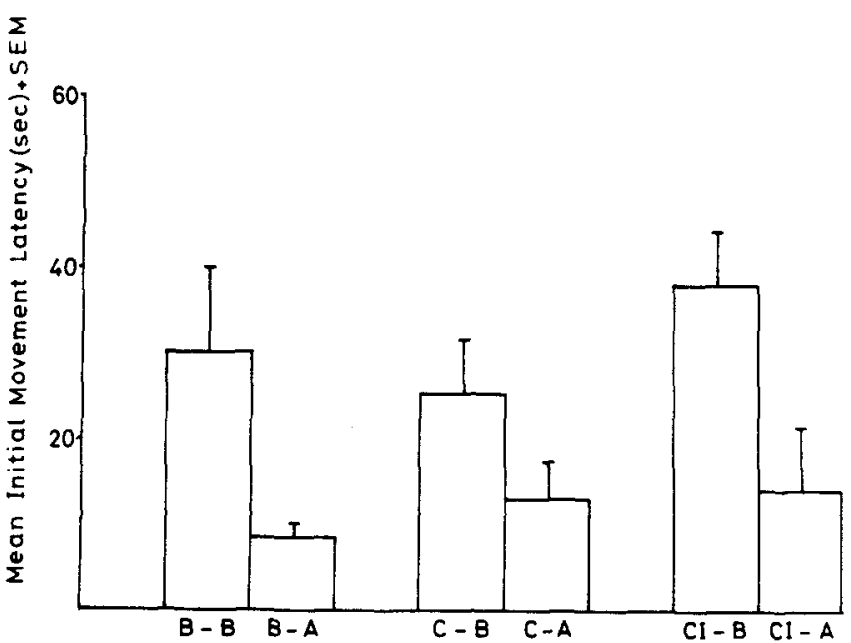

FIG. 2. Latency to initial motor activity in an open field after acute and chronic stress and imipramine (mean sec+SEM). Figure legend presented in Fig. 1.

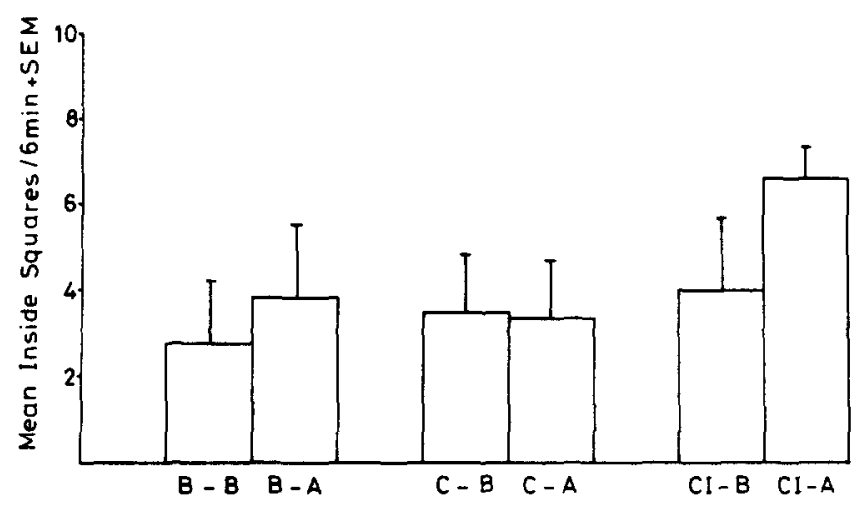

FIG. 3. Center field penetration in an open field in the rat. Effects of acute and chronic stress and imipramine (mean+SEM). Figure legend presented in Fig. 1.

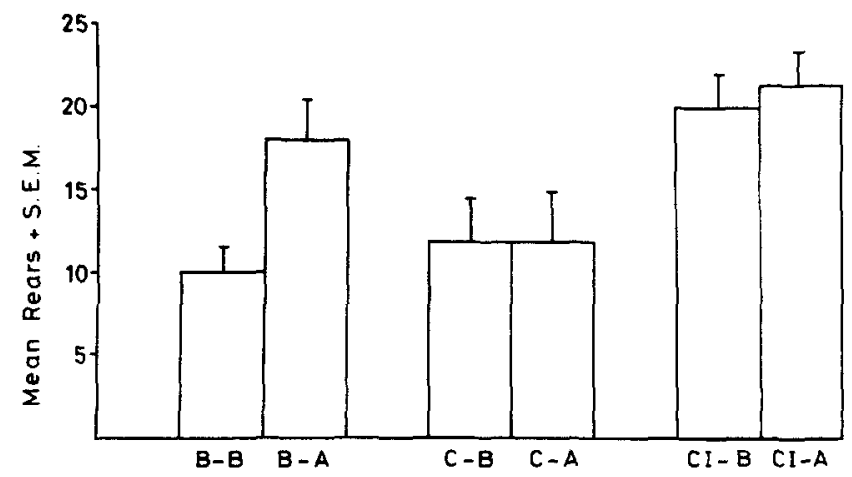

FIG. 4. Effects of acute and chronic stress and imipramine upon rearing activity in an open field (mean+SEM). Figure legend presented in Fig. 1. 


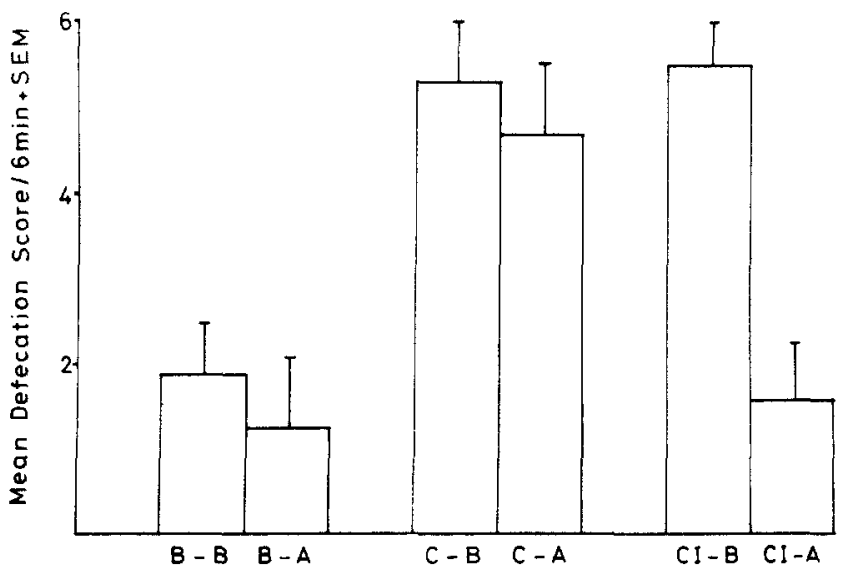

FIG. 5. Defecation score (mean bolus + SEM) in an open field. Effects of acute and chronic stress and imipramine. Figure legend in Fig. 1.

Defecation Score 2

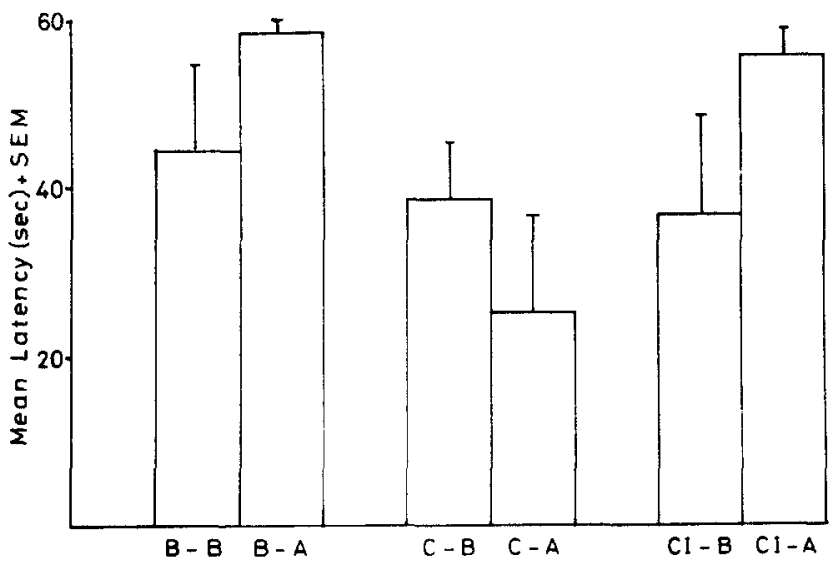

FIG. 6. Defecation score (latency to initial defecation) in an open field (mean sec+SEM). Effects of acute and chronic stress and imipramine. Figure legend in Fig. 1.

those seen in motor activation. There was an overall groups' effect, $F(5,42)=5.1, p<0.001$. Chronic stress increased defecation in the unstressed condition: in the acutely stressed groups, the saline treated chronic stress group defecated more than controls and this was reversed by imipramine treatment. The imipramine treated group showed a significant decrease in defecation between unstressed and acutely stressed conditions.

A further measure of defecation (latency: Fig. 6) followed a converse course. There was an overall significant groups effect, $\mathrm{F}(5,42)=4.0, p<0.005$. Chronic stress lowered the latency to defecation in the unstressed groups but only the saline treated chronically stressed group was significantly lower than controls in the acute stressed condition.

Results for plasma corticosterone are presented in Fig. 7. Groups differed overall from each other, $F(8,60)=10.4$, $p<0.0001$. This represented both an elevation in corticoste-

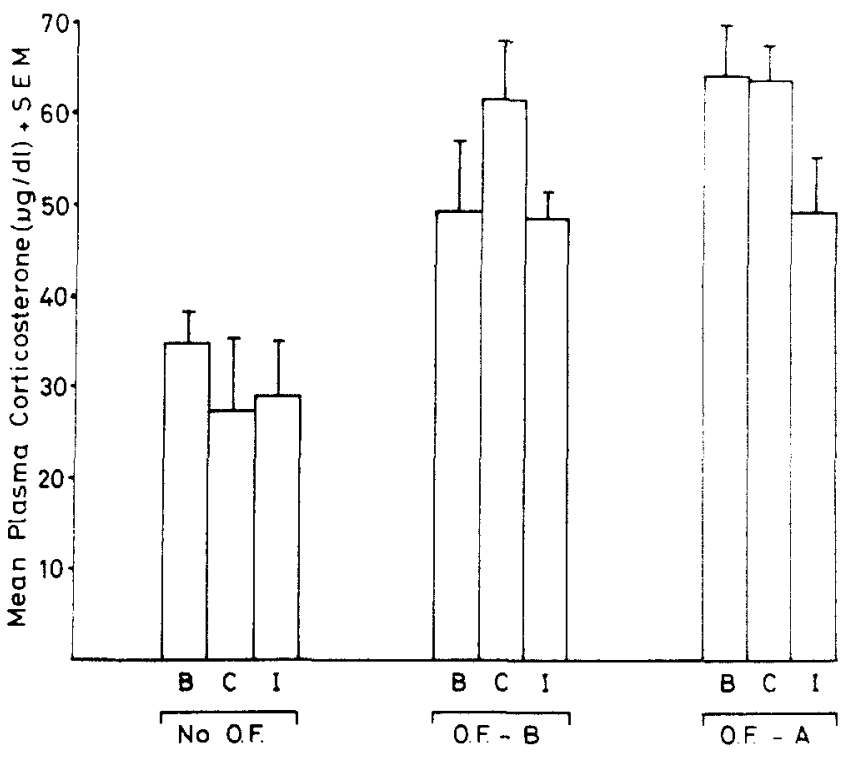

FIG. 7. Endocrine activity in response to acute and chronic stress. imipramine, and open field testing. Mean plasma corticosterone value in $\mu \mathrm{g} / \mathrm{dl}+\mathrm{SEM}$. NO $\mathrm{OF}=$ never exposed to open field; $O F$ $\mathrm{B}=$ basal open field exposure prior to sacrifice; $\mathrm{OF} A=$ exposed to 1 hr noise stress and open field prior to sacrifice. $B=$ basal (no chronic stress); $C=$ exposed to chronic stress regimen; $I=$ exposed to both chronic stress and concurrent imipramine.

rone due to open field testing, a marginal $(p<0.06)$ further increase due to stress prior to the open field, a significant increase in corticosterone in stressed subjects vs basal subjects (i.e., open field groups), and a significant imipramine induced reduction in plasma corticosterone in the chronically stressed groups. It must be noted that a slight nonsignificant decrease in corticosterone was present after chronic stress in those rats which were not behaviorally tested.

Figure 8 confirms the above findings based upon the summary measure. The overall effect was significant, $F(5,35)=4.1, p<0.005$. Post hoc comparisons confirmed the major findings that acute stress produced behavioral activation, a loss of stress induced activation by chronic stress, and a restoration of acute stress responding after imipramine treatment.

\section{DISCUSSION}

One question inherent in the development of preclinical procedures concerns the systematic validity of a given model. While explicit criteria exist for evaluating laboratory models, they are often utilized selectively or inconsistently. At the very least, however, a commonality of behavioral phenomenology (depressive behavior upon the part of the animal), physiology (the existence of similar indicators of a central disorder insofar as preexisting physiological differences allow comparison) and of pharmacological response must be addressed by the serious researcher. The present study presents both behavioral and physiological evidence which confirm a previous report [5] and also suggest that therapeutic response is not limited to inhibitors of 


\section{Activity Score}

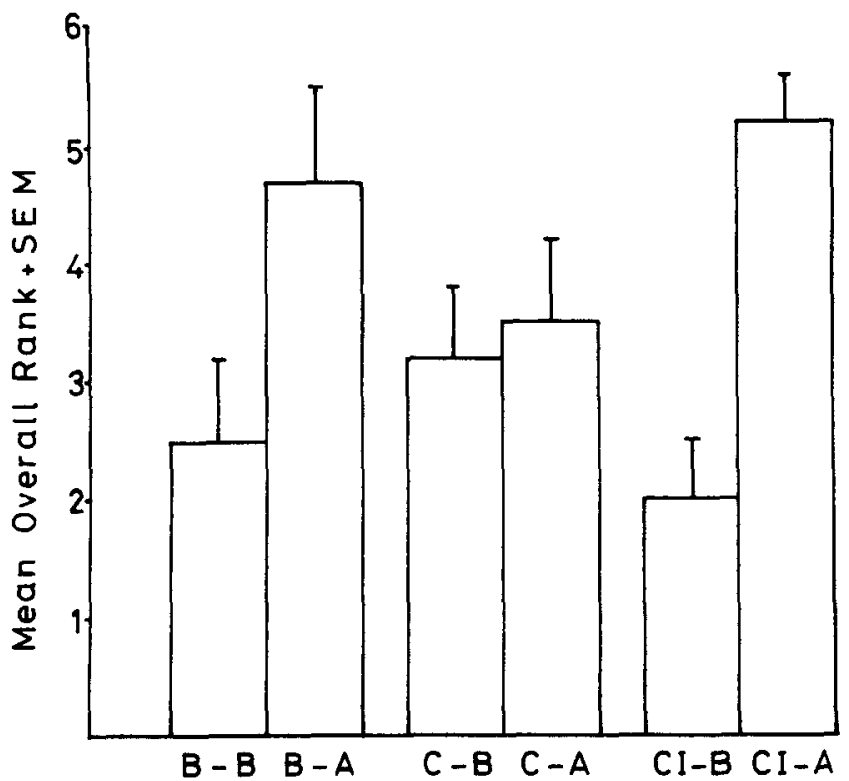

FIG. 8. Summary measure of overall activity based upon mean rank across categories. For a detailed description of the measure and its computation see text (Method). Figure legend as in Fig. 1.

monoamine oxidase, but may be present with tricyclic antidepressants. This response is probably not limited to drug classes thought to act via their noradrenergic effects, since other tricyclics (e.g., amitriptyline) and tetracyclics (e.g., mianserine) are equally effective.

Acute stress induced alterations in activity were seen in five categories of behavior (activity, latency, center field activity, defecation scores). The acute stress effect was clearly blunted by a history of antecedent stress, and restored by drug treatment. Antidepressants such as imipramine produced no consistent effects upon the acute stress response in rats not exposed to chronic stress procedures.

Within limits, concomitant psychoendocrine measures further suggest similarities to clinical disorders. A number of pituitary-adrenal response comparisons were marginally significant. This must be understood, however, as being due in part to a more protracted testing interval than previously employed. Open field exposure is mildly stressful, and therefore may have reduced effects due to an overall increase in baseline endocrine activity.

Perhaps the most puzzling aspect of the endocrine data is the apparent absence of a hypersecretion of corticosterone in chronically stressed but behaviorally naive rats. This may point to a potential difference from clinical pathophysiology. On the other hand open field tested rats did show a typical basal hypersecretion and may in fact embody a greater similarity to typical clinical conditions then behaviorally naive unstressed rats. When psychoendocrine studies are carried out clinically the presence of additional staff and the drawing of blood may both be novel and mildly stressful, as is the open field for the rat. Finally, Carroll [2] has reported no effect of acute stress on plasma cortisol in human depressives. This may be related to the acute stress conditions of the present design (rightmost three columns of Fig. 7) which may be seen to not show an effect of chronic stress.

It is of particular interest that in the present study imipramine, a drug with established psychomotor inhibitory properties (e.g. $[4,8]$; also the present study) nonetheless restored a stress induced activation deficit. Clearly this cannot be regarded as a mere summation of behavioral and pharmacological excitation, since the drug itself is normally inhibitory. This finding suggests that the facilitatory effect is highly specific and dependent upon the prior existence of behavioral depression.

The precise mechanisms underlying these effects remain to be identified. Some effects, particularly defecation, may be due to the effects of imipramine upon gastric motility, although it is not clear this explanation also accounts for other behavioral effects, the consistency seen across behaviors, or the general consistency across drug categories. Another possibility rests with the notion of "stress tolerance." Weiss and co-workers have reported some reduction of acute stress-responsiveness after a history of stress [12]. Although stress modality and chronic stress procedures differ, it is possible that the same or analogous processes are involved in the present behavioral reductions. The present study extends previous studies upon a novel animal model of depression, and suggests a useful procedure for further examining questions of clinical interest.

\section{ACKNOWLEDGEMENTS}

Supported by grant MH31588 to Dr. B. J. Carroll (with Dr. Katz as co-investigator). Statistical assistance of Bob Shea and technical assistance of Pam Olton are acknowledged with gratitude. Dr. Katz currently is a Sloan Research Foundation Fellow in Neuroscience, Kevin Roth currently is a predoctoral fellow within the laboratory of Dr. J. Barchas. Correspondence to the second author.

\section{REFERENCES}

1. Beck, A. T. Depression, Clinical Experimental and Theoretical Aspects. New York: Hoerber Med. Div. Harper and Row, 1967.

2. Carroll, B. J. Studies with hypothalamic pituitary adrenal stimulation tests in depression. In: Depressive I/lness Some Research Studies, edited by B. M. Davies, B. J. Carroll and R. M. Mowbray. Springfield, III.: Charles C. Thomas Publisher, 1972, pp. 149-201.

3. Dixon, W. J. and F. J. Massey. Introduction to Statistical Analysis. New York: McGraw Hill, 1969.
4. Furgiuele, A. R., M. H. Aumente and Z. P. Horovitz. Acute and chronic effects of imipramine and desipramine in normal rats and in rats with lesioned amygdalae. Archs int. Pharmacodyn. 151: $129,1964$.

5. Katz, R. J. Animal models and human depressive disorders. Neurosci. Biobehav. Rev. 5: 231-246, 1981.

6. Katz, R. J., K. A. Roth and B. J. Carroll. Acute and chronic stress effects on open field activity in the rat: Implications for a model of depression. Neurosci. Biobehav. Rev. 5: 247-251, 1981. 
7. Katz, R. J. and S. Hirsch. Amitriptyline and scopolamine in an animal model of depression. Neurosci. Biobehav. Rev. 5: 265$271,1981$.

8. Garzon, J., J. A. Fuentes and J. Delrio. Antidepressants selectively antagonize the hyperactivity induced in rats by long term isolation. Eur. J. Pharmac. 593: 93-296, 1979.

9. Porsolt, R. D., G. Arton, N. Biaret, M. Daniel and M. Jalfre. Behavioral despair in rats, a new model sensitive to antidepressant treatments. Eur. J. Pharmac. 47: 379-391, 1978.
10. Murphy, B. E. P. Some studies of the protein binding of steroids and their application to the routine micro and ultramicro measurement of various steroids in body fluids by competitive protein-binding radioassay. J. clin. Endocr. 27: 973-990, 1967.

11. Von Praag, H. H. Psychotropic Drugs-A Guide for the Practitioner. New York: Brunner-Mazel, 1978.

12. Weiss, J. M., H. I. Glaser, L. A. Pohorecky, J. Brick and N. E. Miller. Effects of chronic exposure to stressors on avoidanceescape behaviors and on brain-norepinephrine. Psychosom. Med. 37: 522-534, 1975. 